\title{
Suppression of arbitrary internal coupling in a quantum register
}

\author{
Marcus Stollsteimer and Günter Mahler \\ Institut für Theoretische Physik, Universität Stuttgart, Pfaffenwaldring 57, 70550 Stuttgart, Germany
}

(November 3, 2018)

\begin{abstract}
For the implementation of a quantum computer it is necessary to exercise complete control over the Hamiltonian of the used physical system. For NMR quantum computing the effectively acting Hamiltonian can be manipulated via pulse sequences. Here we examine a register consisting of $N$ selectively addressable spins with pairwise coupling between each spin pair. We show that complete decoupling of the spins is possible independent of the particular form of the spin-spin interaction. The proposed method based on orthogonal arrays is efficient in the sense that the effort regarding time and amount of pulses increases only polynomially with the size $N$ of the register. However, the effect of external control errors in terms of inaccurate control pulses eventually limits the achievable precision.
\end{abstract}

03.67.Lx, 76.60.-k

\section{INTRODUCTION}

Couplings within a quantum register may be considered a necessary evil: the two-qubit operations needed for the realization of quantum computing [1] rely on interactions between different qubits. For fast gate operations even strong couplings are desirable. On the other hand, residual interactions, that are always present, disturb the system and lead to unwanted evolution, so that stored information may get lost. This kind of "hardware" problems exist even in situations where external (classical) contacts are supposed to control pertinent parameters of the network Hamiltonian [2,3]. They are particularly relevant for conventional NMR implementations [4.5], where couplings cannot simply be turned on and off, but are predetermined by the respective Hamiltonian.

The interplay between local disorder and interactions has recently been investigated with respect to the possible emergence of the so-called "quantum chaos" [6]. As expected, disorder helps to avoid the delocalization of local register states. On the other hand, (static) disorder itself presents a kind of control loss, which is not welcome. We will include some aspects thereof via faulty gate operations.

Fortunately, by using sophisticated pulse sequences a wide range of control can be exerted on the system. Coupling terms can be removed in an NMR spin echo experiment by applying so-called refocusing pulses, essentially reversing the time evolution. This method can be extended to the task of removing selected or even all coupling terms in a quantum register of $N$ qubits. Such a scheme has been described by Linden et al. [7]. Unfortunately it lacks efficiency. Efficient schemes have been proposed by Leung et al. [8] and Jones and Knill [9], respectively. However, these studies focus on the special model of weak scalar coupling only. For other coupling models it remains open whether there exist generalized schemes, let alone efficient ones. In this paper we will demonstrate that, indeed, any interaction can be suppressed efficiently, regardless of the specific coupling model, and even without knowledge of the underlying Hamiltonian.

Perfect decoupling in general requires fast application of many pulses. In a real implementation the pulses will never be ideal, which will impair the decoupling. Quantum error correction methods could be used, but they require supplementary resources in the form of auxiliary qubits. Here, we want to probe the limitations to the given method that arise from faulty gate operations without considering error correction methods.

\section{MODEL AND AVERAGE HAMILTONIAN}

We consider a system of $N$ coupled spin- $1 / 2$ particles (qubits) with the general Hamiltonian model

$$
\mathcal{H}=\sum_{\mu=1}^{N} \sum_{i=1}^{3} \omega_{i}^{(\mu)} \hat{\sigma}_{i}^{(\mu)}+\sum_{\mu<\nu}^{N} \sum_{i, j=1}^{3} H_{i j}^{\mu \nu} \hat{\sigma}_{i}^{(\mu)} \otimes \hat{\sigma}_{j}^{(\nu)}
$$

where the $\hat{\sigma}_{i}(i=1,2,3$ or $x, y, z)$ denote the Pauli operators. The terms linear in those are the Zeeman terms specified by the 3 -dimensional vectors $\boldsymbol{\omega}^{(\mu)}=\left(\omega_{i}^{(\mu)}\right)$. The interaction between two qubits $\mu$ and $\nu$ is described by the coupling tensor $\mathrm{H}^{\mu \nu}=\left(H_{i j}^{\mu \nu}\right)$. We will call couplings "diagonal" when they are represented by a diagonal coupling tensor $\mathrm{H}=\operatorname{diag}\left(J_{x}, J_{y}, J_{z}\right)$. Examples are the strong and weak scalar coupling, with $J_{x}=J_{y}=J_{z}=: J$ and $J_{x}=J_{y}=0$, respectively.

We will now subject the system to a cyclic pulse train. The pulses are assumed to be infinitely short, so that they can be represented by instantaneous operations $\hat{P}_{k}$. Each pulse is followed by a free evolution period of duration $\tau_{k}$. A sequence consisting of $n$ pulses will thus be denoted by

$$
\left(\hat{P}_{1}, \tau_{1}, \ldots, \hat{P}_{n}, \tau_{n}\right)
$$

where the pulses are applied from left to right. For the further analysis we use the formalism of average Hamiltonian (AH) theory 10 12]. The time evolution operator is given by $\hat{U}\left(t_{c}\right)=\prod_{k=1}^{n} e^{-\mathrm{i} \mathcal{H} \tau_{k}} \hat{P}_{k}$, where $t_{c}:=\sum_{k=1}^{n} \tau_{k}$ is the cycle time (all products are ordered 
with increasing $k$ from right to left). For a cyclic sequence (defined by $\prod_{k=1}^{n} \hat{P}_{k}=\hat{1}$ ), we can write this as $\hat{U}\left(t_{c}\right)=\prod_{k=1}^{n}\left[\left(\prod_{l=1}^{k} \hat{P}_{l}\right)^{-1} e^{-\mathrm{i} \mathcal{H} \tau_{k}}\left(\prod_{l=1}^{k} \hat{P}_{l}\right)\right]$. Using the identity $\hat{A}^{-1} \exp (\hat{B}) \hat{A}=\exp \left(\hat{A}^{-1} \hat{B} \hat{A}\right)$ we get

$$
\hat{U}\left(t_{c}\right)=\prod_{k=1}^{n} e^{-\mathrm{i} \mathcal{H}_{k} \tau_{k}} .
$$

The operators $\mathcal{H}_{k}$ are the Hamiltonians in the so-called "toggling frame" 11

$$
\mathcal{H}_{k}:=\hat{Q}_{k}^{-1} \mathcal{H} \hat{Q}_{k}, \quad \text { with } \hat{Q}_{k}:=\prod_{l=1}^{k} \hat{P}_{l} .
$$

By expanding the exponential in (2.3) and collecting terms of equal order in $\mathcal{H}_{k} \tau_{k}$ the time evolution operator can be written as

$$
\hat{U}\left(t_{c}\right)=e^{-\mathrm{i}\left(\overline{\mathcal{H}}+\overline{\mathcal{H}}^{(1)}+\overline{\mathcal{H}}^{(2)}+\cdots\right) t_{c}},
$$

where the operators $\overline{\mathcal{H}}, \overline{\mathcal{H}}^{(1)}, \ldots$ are the average Hamiltonians of increasing order. In the remainder of this paper we will only use pulse sequences consisting of equally spaced pulses $\left(\tau_{k}=\tau\right)$. For such a sequence with $n$ pulses (" $n \tau$-sequence") the leading order terms of the $\mathrm{AH}$ expansion are given by

$$
\begin{aligned}
\overline{\mathcal{H}} & =\frac{1}{n} \sum_{k=1}^{n} \mathcal{H}_{k}, \\
\overline{\mathcal{H}}^{(1)} & =-\frac{\mathrm{i} t_{c}}{2 n^{2}} \sum_{j>k}\left[\mathcal{H}_{j}, \mathcal{H}_{k}\right] .
\end{aligned}
$$

(For a more detailed derivation refer to App. B of [12].) The time evolution can therefore be described approximately by the average of the toggling frame Hamiltonians, $\overline{\mathcal{H}}$, in the following simply referred to as "the average Hamiltonian". This holds exactly for vanishing commutators between all $\mathcal{H}_{k}$.

For a given time period $k$, the pulse sequence will lead to the transformation $\hat{Q}_{k}=\bigotimes_{\mu=1}^{N} \hat{Q}_{k}^{(\mu)}$, where $\hat{Q}_{k}^{(\mu)}$ is the transformation on qubit $\mu$. The applied pulses, and hence the operators $\hat{Q}_{k}^{(\mu)}$, are unitary transformations. Any unitary transformation in $S U(2)$ can be represented by ordinary 3 -dimensional rotation matrices $\mathrm{R}=\left(R_{i j}\right)$ via the relation

$$
\hat{Q}^{\dagger} \hat{\sigma}_{i} \hat{Q}=\sum_{j=1}^{3} R_{i j} \hat{\sigma}_{j},
$$

so that any pulse sequence can be characterized by a set of matrices $\mathrm{R}_{k}^{(\mu)}$.

The resulting average Hamiltonian can be written in the same form as the original system Hamiltonian (2.1); it is straightforward to verify from (2.4) and (2.8) that the vector representing the Zeeman part for qubit $\mu$ is given by

$$
\overline{\boldsymbol{\omega}}^{(\mu)}=\frac{1}{n} \sum_{k=1}^{n} \boldsymbol{\omega}_{k}^{(\mu)}=\frac{1}{n} \sum_{k=1}^{n}\left(\mathrm{R}_{k}^{(\mu)}\right)^{T} \boldsymbol{\omega}^{(\mu)},
$$

and the $3 \times 3$ tensor representing the coupling part for a given pair $\mu, \nu$ by

$$
\overline{\mathrm{H}}^{\mu \nu}=\frac{1}{n} \sum_{k=1}^{n} \mathrm{H}_{k}^{\mu \nu}=\frac{1}{n} \sum_{k=1}^{n}\left(\mathrm{R}_{k}^{(\mu)}\right)^{T} \mathrm{H}^{\mu \nu} \mathrm{R}_{k}^{(\nu)} .
$$

Our goal is to effectively remove all interactions by applying a proper pulse sequence. The more general task of generating an evolution corresponding to selected couplings being turned on or off requires only minor modifications. Specifically, we want to achieve "first order decoupling", meaning that the average Hamiltonian vanishes. For now higher order terms in the AH expansion will be neglected. Also, the Zeeman interactions shall be excluded for the time being.

\section{DECOUPLING FOR 2-QUBIT NETWORK}

We consider at first the case of two qubits. Ignoring Zeeman interactions we are left with a single interaction term represented by the tensor $\mathrm{H}^{12}=: \mathrm{H}$. First order decoupling is then achieved by a pulse sequence resulting in $\overline{\mathrm{H}}=0$.

Two principally different kinds of pulses can be used, spin-selective and nonselective ones. From (2.10) the following theorem on the selectivity of the required decoupling pulses can be derived:

Theorem 1 A coupling represented by a tensor $\mathrm{H}$ with $\operatorname{Tr} \mathbf{H} \neq 0$ cannot be decoupled with nonselective pulses.

Proof: For nonselective pulses $\left(\mathrm{R}_{k}^{(1)}=\mathrm{R}_{k}^{(2)}\right)$ the toggling frame coupling tensor is an orthogonal transformation of the original coupling tensor, therefore $\operatorname{Tr} \mathrm{H}_{k}=\operatorname{Tr} \mathrm{H}=$ $\operatorname{Tr} \overline{\mathrm{H}}$. For decoupling one needs $\overline{\mathrm{H}}=0$, and hence $\operatorname{Tr} \mathrm{H}$ must vanish. (This also holds for sequences with different $\tau_{k}$, where (2.10) is then given by a weighted average.)

In the case of, e.g., direct dipolar couplings (with $\mathbf{H} \propto \operatorname{diag}(1,1,-2)$ in the high-field approximation) this condition is fulfilled and nonselective sequences like the well-known WHH-4 [13] can be applied. On the other hand, the widely discussed strong or weak scalar coupling requires selective pulses.

We now give a specific first order decoupling sequence for arbitrary couplings using spin-selective $\pi$ pulses with respect to the coordinate axes $x, y, z$. These pulses are given by the operators $\hat{X}=e^{-\mathrm{i} \pi \hat{\sigma}_{x} / 2}=\mathrm{i} \hat{\sigma}_{x}$, and analogously for $\hat{Y}$ and $\hat{Z}$. The corresponding rotation matrices are 


$$
\begin{aligned}
& X=\mathrm{R}_{x}=\operatorname{diag}(+1,-1,-1), \\
& Y=\mathrm{R}_{y}=\operatorname{diag}(-1,+1,-1), \\
& Z=\mathrm{R}_{z}=\operatorname{diag}(-1,-1,+1),
\end{aligned}
$$

with the multiplication table shown in Table II. The decoupling is based on the following property

$$
a_{0} I+a_{1} X+a_{2} Y+a_{3} Z=0 \quad \Leftrightarrow \quad a_{i}=a,
$$

where $I$ denotes the identity matrix. A weighted sum of the matrices $I, X, Y, Z$ vanishes if and only if the weight factors are equal.

Following [8] a pulse sequence will be written as a matrix of transformations $I, X, Y$, and $Z$ specifying the corresponding $\hat{Q}$ or R on a specific qubit for a certain time interval of duration $\tau$. The $n$ time intervals correspond to the columns (from left to right) and the different qubits $\mu=1,2, \ldots, N$ to different rows. We may thus speak of an $n$-dimensional row vector $\boldsymbol{R}^{(\mu)}$, the elements of which are the ordered sequence of transformation matrices for given $\mu, \mathrm{R}_{k}^{(\mu)}, k=1,2, \ldots, N$. This representation gives more direct insight into what is happening to the coupling Hamiltonian than the notation involving the actually applied pulses.

Now consider the sequence

$$
\left(\begin{array}{l}
\boldsymbol{R}^{(1)} \\
\boldsymbol{R}^{(2)}
\end{array}\right)=\left(\begin{array}{cccc}
I & I & I & I \\
I & X & Z & Y
\end{array}\right)
$$

which can be implemented by the following train of $\pi$ pulses on qubit 2 [cf. (2.2)]

$$
\left(\tau, \hat{X}^{(2)}, \tau, \hat{Y}^{(2)}, \tau, \hat{X}^{(2)}, \tau,-\hat{Y}^{(2)}\right) .
$$

(The final $y$-pulse is applied in order to make the sequence cyclic.) The resulting average Hamiltonian is given by

$$
\overline{\mathrm{H}}=\frac{1}{n} \mathrm{H} \sum_{k} \mathrm{R}_{k}^{(2)}=\frac{1}{n} \mathrm{H}(I+X+Y+Z),
$$

which vanishes due to (3.2) for any coupling tensor $\mathrm{H}$. This sequence thus effectively removes an arbitrary coupling between two qubits. The Hamiltonian does not even have to be known, because the same sequence works for any interaction. Hence it follows:

Theorem 2 Any interaction between two spins can be decoupled to first order using a sequence of spin-selective pulses.

For diagonal interaction models 2.10 becomes

$$
\overline{\mathrm{H}}=\frac{1}{n} \mathrm{H} \sum_{k=1}^{n} \mathrm{R}_{k}^{(1)} \mathrm{R}_{k}^{(2)}=: \frac{1}{n} \mathrm{H} \sum_{k=1}^{n} \mathrm{R}_{k}^{(12)},
$$

because all matrices are diagonal (for the $\pi$ pulses under investigation). Then it does not matter how the transformations are "distributed" between the two qubits, because only the product needs to be considered. Thus alternative sequences are possible, which will be particularly interesting for the $N>2$ case discussed later. The sequence

$$
\left(\begin{array}{l}
\boldsymbol{R}^{(1)} \\
\boldsymbol{R}^{(2)}
\end{array}\right)=\left(\begin{array}{cccc}
I & X & X & I \\
I & I & Y & Y
\end{array}\right)
$$

leads to the same transformations of the coupling tensor as (3.3) and thus also decouples the interaction. Furthermore, it has the additional feature of removing the Zeeman terms, for the typical case that they only consist of a $z$-component, $\omega_{z} \hat{\sigma}_{z}$. The corresponding pulses applied both on qubit 1 and 2 are given by

$$
\left(\tau, \hat{X}^{(1)}, \tau, \hat{Y}^{(2)}, \tau, \hat{X}^{(1)}, \tau, \hat{Y}^{(2)}\right)
$$

The sequential order of the transformations clearly has no influence on $\overline{\mathrm{H}}$ and thus on the first order decoupling. For the above mentioned sequences it is chosen so that no $z$-pulses are needed, which usually cannot be implemented in conventional NMR. The higher order terms, however, are affected by the order.

\section{DECOUPLING FOR $N$-QUBIT NETWORK}

We now turn to a system consisting of $N$ qubits. It often suffices to take only nearest and, possibly, next nearest neighbors into account. We will come back to this issue later. For now we discuss the most general case of a completely coupled system, i. e. each qubit is coupled to all the other ones. From 2.10 it is evident that each pair interaction $\mathrm{H}^{\mu \nu}$ can be treated separately, essentially leading back to the $N=2$ case. So, we can use the sequence for $N=2$ as a starting point. An $n \tau$ decoupling sequence for $N$ qubits will be represented by an $N \times n$ matrix. Decoupling means elimination of all $\mathrm{H}^{\mu \nu}$. The task is to find a matrix that yields a valid $N=2$ decoupling sequence for any pairing of rows.

\section{A. Simple Scheme}

The simplest approach is to recursively nest the sequence (3.3) in analogy to the method in [7]. When a qubit is added to a valid sequence, it will be exposed to a complete unit cycle during each time interval of the initial sequence. For 3 qubits we thus get:

$$
\left(\begin{array}{llllllllllllllll}
I & I & I & I & I & I & I & I & I & I & I & I & I & I & I & I \\
I & I & I & I & X & X & X & X & Z & Z & Z & Z & Y & Y & Y & Y \\
I & X & Z & Y & Y & Z & X & I & I & X & Z & Y & Y & Z & X & I
\end{array}\right) .
$$

While the additional qubit will undergo one $4 \tau$ unit cycle, the transformations on the other qubits are constant, and it will therefore decouple. However, this scheme is not efficient in terms of the time intervals $\tau$ and pulses needed, which both scale as $4^{N-1}$ because the sequence 
has to be prolonged by a factor 4 for each added qubit. The advantage is that at each time interval only one pulse has to be applied. This is why every second unit cycle is reversed. Furthermore, the almost realized mirror symmetry $\left(\mathcal{H}_{k}=\mathcal{H}_{n+1-k}\right)$ is expected to reduce the higher order correction terms: for a symmetric sequence, all correction terms of odd order vanish [12].

\section{B. Efficient Scheme Using Orthogonal Arrays}

Much more efficient schemes can be constructed using the notion of orthogonal arrays $(O A)$, which have been extensively studied in the context of combinatorics, design theory, and error-correcting codes. A comprehensive treatment can be found in [14]. First we want to give a definition of orthogonal arrays and then demonstrate how they are related to the decoupling problem.

Let $S$ be a set of $s$ symbols. Then an orthogonal array is defined by

Definition $1 A k \times \lambda s^{t}$ array $A$ with entries from $S$ is said to be an orthogonal array with $s$ levels, strength $t$, and index $\lambda(0 \leq t \leq k)$ if every $t \times \lambda s^{t}$ subarray of $A$ contains each $t$-tuple based on $S$ exactly $\lambda$ times as a column. It will be denoted by $O A\left(\lambda s^{t}, k, s, t\right)$.

For our purposes we will only regard the case $t=2$ and $s=4$. Usually the symbols are chosen as the numbers 0 , $1,2,3$. We will interpret them as the $\pi$ rotation matrices including the identity matrix, so that $S=\{I, X, Y, Z\}$. As an example regard the following $O A(16,5,4,2)$

$$
\left(\begin{array}{llllllllllllllll}
I & I & I & I & X & X & X & X & Y & Y & Y & Y & Z & Z & Z & Z \\
I & X & Y & Z & I & X & Y & Z & I & X & Y & Z & I & X & Y & Z \\
I & X & Y & Z & X & I & Z & Y & Y & Z & I & X & Z & Y & X & I \\
I & X & Y & Z & Y & Z & I & X & Z & Y & X & I & X & I & Z & Y \\
I & X & Y & Z & Z & Y & X & I & X & I & Z & Y & Y & Z & I & X
\end{array}\right) .
$$

If you pick any two rows (e.g. row 2 and 5), each of the $s^{t}=16$ possible 2 -dimensional column vectors appears exactly once, hence $\lambda=1$. Definition 1 implies further that every row contains each symbol exactly $\lambda s=4$ times. As it turns out, these properties are sufficient for the array to be a valid decoupling sequence:

Theorem 3 Any $O A(16 \lambda, k, 4,2)$ can be used to decouple $k+1$ qubits within $n=16 \lambda$ time intervals.

Proof: Pick two rows $\mu$ and $\nu$ of the $O A$ and consider the corresponding average Hamiltonian according to (2.10), $\overline{\mathrm{H}}^{\mu \nu}=\frac{1}{n} \sum_{j=1}^{n} \mathrm{R}_{j}^{(\mu)} \mathrm{H}^{\mu \nu} \mathrm{R}_{j}^{(\nu)}, n=16 \lambda$. Each of the $s^{t}=$ 16 combinations $\left(\mathrm{R}_{j}^{(\mu)}, \mathrm{R}_{j}^{(\nu)}\right)=(I, I),(I, X), \ldots,(Z, Z)$ occurs exactly $\lambda$ times. We thus get $\overline{\mathrm{H}}^{\mu \nu}=\frac{1}{16}(I+X+Y+$ $Z) \mathrm{H}^{\mu \nu}(I+X+Y+Z)$, which vanishes for any $\mathrm{H}^{\mu \nu}$. We can further add an additional $(k+1)$ th row to the array, consisting only of I's. As every row of the OA contains an equal number $\lambda$ s of each symbol, the $A H$ involving the $(k+1)$ th qubit is $\overline{\mathrm{H}}^{\mu, k+1}=\frac{1}{4} \mathrm{H}^{\mu, k+1}(I+X+Y+Z)=0$. Thus, the $O A$ can be used to decouple $k+1$ qubits.
Using large enough $O A \mathrm{~s}(k+1 \geq N)$, decoupling sequences for an arbitrary number $N$ of qubits can thus be constructed. We note that simultaneous pulses on different qubits are necessary for this scheme to work. The essential question is for which sets of parameters $\lambda, k, s, t$ an $O A$ exists at all. Specifically, we want to find arrays with $k$ as large as possible, in order to decouple many qubits with the least effort.

Bose and Bush [15] have given a recursive construction scheme for arrays of strength 2, providing $O A$ s with large values $k$. From their Theorem 4 follows the existence of all $O A(n, k, 4,2)$ with

$$
n=2^{u+4}, \quad k=\left\{\begin{array}{ll}
\frac{1}{3}(n-1), & u=0,2,4, \ldots . \\
\frac{1}{3}(n-5), & u=1,3,5, \ldots .
\end{array} .\right.
$$

An example of the underlying construction scheme will be given in the appendix. This guarantees the existence of the arrays $O A(16,5,4,2), O A(32,9,4,2), O A(64,21,4,2)$, $O A(128,41,4,2)$, and so on. [Note that this construction does not yield all possible $O A$ s, there exists, for example, an $O A(48,13,4,2)$.] From (4.3) we can derive a lower bound on the efficiency of the scheme: An $\left(n=2^{u+4}\right) \tau$ sequence can be used to decouple up to $N=\frac{1}{3}(n \pm 2)$ qubits (the sign depending on $u$ being even or odd). It follows that $N$ qubits $(N \geq 3)$ can be decoupled by a sequence consisting of $n=c N-2$ time intervals, where $3 \leq c \leq 6$. The number of pulses that are needed for the sequence is less than $N(c N-2)$. Hence it is possible to decouple (to first order) any interaction with linearly increasing effort in time: the scheme is efficient.

\section{Efficient Scheme for Diagonal Couplings}

For diagonal couplings there exists an even more efficient scheme. When the transformations on two qubits $\mu$ and $\nu$ are given by the row vectors $\boldsymbol{R}^{(\mu)}$ and $\boldsymbol{R}^{(\nu)}$ [cf. (3.3)], the average Hamiltonian is, in analogy to (3.6),

$$
\overline{\mathrm{H}}^{\mu \nu}=\frac{1}{n} \mathrm{H}^{\mu \nu} \sum_{k=1}^{n} \mathrm{R}_{k}^{(\mu)} \mathrm{R}_{k}^{(\nu)}=: \frac{1}{n} \mathrm{H}^{\mu \nu}\left(\boldsymbol{R}^{(\mu)} \cdot \boldsymbol{R}^{(\nu)}\right) .
$$

It vanishes whenever the "scalar" product $\boldsymbol{R}^{(\mu)} \cdot \boldsymbol{R}^{(\nu)}$ (which is really tensor valued) equals zero. This leads with (3.2) to the following decoupling criterion:

Theorem 4 A pulse sequence represented by a matrix of $N$ rows can be used to decouple $N$ qubits with diagonal coupling between any qubit pair if the scalar products between different row vectors vanish. This is equivalent to the condition that the vector of the element-wise products of two rows contains each of the elements $I, X, Y, Z$ equally often.

The sequences already discussed, like e.g. (4.2), fulfill this condition, but there exist suitable matrices with a smaller number of columns, leading to even shorter 
sequences. These matrices are closely related to the socalled difference schemes or matrices [14], which play an important role in the construction of the $O A$ s discussed in the previous section. Difference schemes are defined over a finite Abelian group of $s$ elements with a binary operation,$+(\mathcal{A},+)$.

Definition 2 An $r \times c$ array $D(r, c, s)$ with entries from $\mathcal{A}$ is called a difference scheme based on $(\mathcal{A},+)$, if for all $\mu$ and $\nu$ with $1 \leq \mu, \nu \leq r, \mu \neq \nu$, the vector difference between the $\mu$ th and $\nu$ th rows contains every element of $\mathcal{A}$ equally often.

Relevant here are difference schemes over the additive group of the Galois field $G F(s)$ (cf. App. A of [14]) with $s=4$ elements:

Theorem $5 A D(r, c, 4)$ over $(G F(4),+)$ is equivalent to a c $\tau$-decoupling sequence for $N=r$ qubits.

Proof: The group of $\pi$ rotations, $(\{I, X, Y, Z\}, *)$, is isomorphic to $(G F(4),+)$, which is evident from Table \. So the elements $I, X, Y, Z$ can be identified with $0,1,2,3$, and the matrix product with the operation + . Each element is its own inverse, so that the difference of two rows equals the sum. Then the defining property for difference schemes is equivalent to the condition of Theorem 4 .

For difference schemes one has $r \leq c$ 16], so that at most $N=n$ qubits can be decoupled with an $n \tau$ sequence. An $r \times r$ array $D(r, r, s)$ is also called generalized Hadamard matrix.

Multiplying a column with a specific element leaves the scalar products invariant, so that the matrices can be brought into a normalized form, with only I's in the first row. The other rows then have to consist of permutations of equal numbers of $I, X, Y, Z$, so that from the outset only sequences with $n=4 u(u \in \mathbb{N})$ time intervals are applicable. By trying all combinations we have found the following matrices for decoupling of up to 4 or 8 qubits, respectively.

$$
M_{4}=\left(\begin{array}{lllll}
I & I & I & I \\
I & X & Y & Z \\
I & Y & Z & X \\
I & Z & X & Y
\end{array}\right),
$$

We will denote such $n \times n$ decoupling matrices corresponding to a $D(n, n, 4)$ by $M_{n}$. We do not show here the also existing $M_{12}$. One is led to suspect that there may exist matrices $M_{n}$ for all $n=4 u, u \in \mathbb{N}$, which, however, is still an open question. But at least for all $n=2^{u}, u \geq 2$, difference schemes can be constructed using the properties of Galois fields [15]. (For a more detailed discussion on the existence of difference schemes see chap. 6 of [14].) A simpler way to find matrices $M_{n}$ can be based on the following theorem:

Theorem 6 Given two decoupling matrices $M_{n_{1}}, M_{n_{2}}$, their direct product $M_{n_{1} n_{2}}=M_{n_{1}} \otimes M_{n_{2}}$ is again a decoupling matrix.

Proof: The $\left(n_{2}(i-1)+k\right)$ th row $\left(i=1, \ldots, n_{1} ; k=\right.$ $\left.1, \ldots, n_{2}\right)$ of the matrix $M_{n_{1} n_{2}},(i, k)$, derives from the ith row vector of $M_{n_{1}}, \boldsymbol{i}$, and the $k$ th row vector of $M_{n_{2}}$, $\boldsymbol{k}$, as $(i, k)=\left(\begin{array}{lllllll}i_{1} k_{1} & \ldots & i_{1} k_{n_{2}} & \ldots & i_{n_{1}} k_{1} & \ldots & i_{n_{1}} k_{n_{2}}\end{array}\right)$. The scalar product between two rows $(i, k)$ and $(j, l)$ is $(i, k) \cdot(j, l)=i_{1} j_{1}(\boldsymbol{k} \cdot \boldsymbol{l})+\cdots+i_{n_{1}} j_{n_{1}}(\boldsymbol{k} \cdot \boldsymbol{l})=(\boldsymbol{i} \cdot \boldsymbol{j})(\boldsymbol{k} \cdot \boldsymbol{l})$. Because all rows in $M_{n_{1}}$ and $M_{n_{2}}$ are orthogonal to each other, $(i, k) \cdot(j, l)$ can only be different from zero for $j=i$, $l=k$. Therefore all rows of $M_{n_{1} n_{2}}$ are orthogonal.

Now using the matrices $M_{4}$ and $M_{8}$ given above one can construct decoupling matrices $M_{2}$ for any $u \geq 2$. Consequently, for $N$ qubits there exists a $(c N) \tau$ decoupling sequence with at most $c N^{2}$ pulses, where $1 \leq c<2$. So, for diagonal couplings the efficiency is further improved compared to the general case of the last section.

\section{Zeeman terms and partially coupled systems}

Removing all Zeeman terms requires only a small modification. From (2.9) it follows that the Zeeman terms of a specific qubit $\mu$ are removed if the transformations on that qubit sum up to zero, $\sum_{k=1}^{n} \mathrm{R}_{k}^{(\mu)}=0$. This corresponds to a vanishing row sum in the matrix of transformations. For the given sequences this is fulfilled for all rows except the first. Thus only the Zeeman interaction of qubit 1 is not removed. So, all Zeeman terms can be suppressed by using a matrix for $N+1$ qubits and omitting the first row.

Similarly, leaving selected couplings intact rather than removing all interaction terms takes only small alterations. To achieve, for example, a time evolution corresponding to one single coupling between qubits 1 and $\mu$, it is merely necessary to use the same transformations on qubit $\mu$ as are used on qubit 1.

In reality the couplings are usually well localized and it is sufficient to take only close neighbors into account. Then much simpler sequences can be used. The situation essentially can be regarded as a coloring problem [9]. For nearest-neighbor couplings the problem reduces to the $N=2$ case, so that 3.3 or 3.7 can be used, subjecting all qubits with odd number to the same transformations and likewise for the even qubits. 


\section{HIGHER ORDER TERMS}

Up to now we have only considered first order decoupling, neglecting the higher order terms in the AH expansion. These terms can be suppressed further by repeating a given sequence with correspondingly shorter time intervals.

Let us regard the sequence we get from repeating $m$ times a given $n \tau$ base sequence during a given cycle time $t_{c}$. It then consists of $m n$ time intervals of duration $t_{c} /(m n)$ each. The average Hamiltonian is the same as for the base sequence and vanishes, while for the first correction term we get from (2.7)

$$
\overline{\mathcal{H}}^{(1)}=-\frac{\mathrm{i} t_{c}}{2 m^{2} n^{2}} \sum_{j>k}^{m n}\left[\mathcal{H}_{j}, \mathcal{H}_{k}\right]
$$

By using the new indices $s=1, \ldots, m$ for the different subcycles and $k=1, \ldots, n$ for the time interval within a given subcycle the sum can be written as $\sum_{j>k}^{m n}\left[\mathcal{H}_{j}, \mathcal{H}_{k}\right]=\sum_{s=s^{\prime}}^{m} \sum_{j>k}^{n}\left[\mathcal{H}_{j}, \mathcal{H}_{k}\right]+$ $\sum_{s>s^{\prime}}^{m} \sum_{j, k}^{n}\left[\mathcal{H}_{j}, \mathcal{H}_{k}\right]=m \sum_{j>k}^{n}\left[\mathcal{H}_{j}, \mathcal{H}_{k}\right]$, implying

$$
\overline{\mathcal{H}}^{(1)}=-\frac{\mathrm{i} t_{c}}{2 m n^{2}} \sum_{j>k}^{n}\left[\mathcal{H}_{j}, \mathcal{H}_{k}\right]
$$

The first correction term is thus reduced by the factor $1 / m$ if the base sequence is repeated $m$ times. In principle, by using arbitrarily fast pulses, perfect decoupling could be realized.

\section{EXAMPLE: SPIN CHAIN}

We will now study a simple example, a spin chain consisting of $N$ spins with a diagonal coupling between nearest neighbors. The corresponding Hamiltonian is [cf. (2.1)]

$$
\begin{aligned}
\mathcal{H}= & -\sum_{\mu=1}^{N} \omega_{z}^{(\mu)} \hat{\sigma}_{z}^{(\mu)}+\sum_{\mu=1}^{N-1}\left(J_{x} \hat{\sigma}_{x}^{(\mu)} \otimes \hat{\sigma}_{x}^{(\mu+1)}\right. \\
& \left.+J_{y} \hat{\sigma}_{y}^{(\mu)} \otimes \hat{\sigma}_{y}^{(\mu+1)}+J_{z} \hat{\sigma}_{z}^{(\mu)} \otimes \hat{\sigma}_{z}^{(\mu+1)}\right) .
\end{aligned}
$$

For this interaction model, the $N=2$ sequence for diagonal couplings (3.7,3.8) can be used (thus $n=4$ ), applying the $x$-pulses on all qubits with odd number and the $y$-pulses on all qubits with even number. The leading correction term $\overline{\mathcal{H}}^{(1)}$ does not vanish, in general: for the respective sum of commutators in 2.7) one gets by induction

$$
\begin{aligned}
\sum_{j>k}^{4}\left[\mathcal{H}_{j}, \mathcal{H}_{k}\right]= & 8 \mathrm{i}\left\{J_{x} J_{y} \sum_{\mu=1}^{N-2}\left(\hat{\sigma}_{x z y}^{(\mu)}+\hat{\sigma}_{y z x}^{(\mu)}\right)\right. \\
& \left.+\sum_{\mu=1}^{N-1}\left(\delta_{\mu u} a_{\mu} \hat{\sigma}_{y x}^{(\mu)}+\delta_{\mu g} b_{\mu} \hat{\sigma}_{x y}^{(\mu)}\right)\right\}
\end{aligned}
$$

with

$$
\begin{aligned}
\hat{\sigma}_{i j}^{(\mu)} & =\hat{\sigma}_{i}^{(\mu)} \otimes \hat{\sigma}_{j}^{(\mu+1)}, \quad \hat{\sigma}_{i j k}^{(\mu)}=\hat{\sigma}_{i}^{(\mu)} \otimes \hat{\sigma}_{j}^{(\mu+1)} \otimes \hat{\sigma}_{k}^{(\mu+2)}, \\
a_{\mu} & =\omega_{z}^{(\mu)} J_{x}+\omega_{z}^{(\mu+1)} J_{y}, \quad b_{\mu}=\omega_{z}^{(\mu)} J_{y}+\omega_{z}^{(\mu+1)} J_{x} .
\end{aligned}
$$

As expected, the correction term vanishes for weak coupling, where only $\hat{\sigma}_{z}$ terms are involved (Ising model, $J_{x}=J_{y}=0$ ) and the time evolution is given exactly by the average Hamiltonian (2.6). We now define the fidelity of a given initial state $|\psi\rangle$ after the time $t_{c}$

$$
F\left(t_{c}\right)=\left|\left\langle\psi\left|\hat{U}\left(t_{c}\right)\right| \psi\right\rangle\right|^{2}
$$

As an example we restrict ourselves to strong scalar coupling (Heisenberg model, $J_{x}=J_{y}=J_{z}=J$ ) and equal chemical shifts $\left(\omega_{z}^{(\mu)}=\omega\right)$; taking as the initial state the state with qubit 1 excited and all other qubits in the ground state, $|\psi\rangle=|100 \ldots 0\rangle$, the fidelity for small $t_{c}$ is approximately

$$
F\left(t_{c}\right) \approx \begin{cases}1-\frac{J^{2} \omega^{2} t_{c}^{4}}{4 m^{2}}, & N=2, \\ 1-\frac{J^{2} t_{c}^{4}}{4 m^{2}}\left[\left(J^{2}+\omega^{2}\right)(N-1)-2 J^{2}\right], & N \geq 3 .\end{cases}
$$

In Fig. 1 the undisturbed evolution is plotted for $N=4$ qubits together with the curves for two decoupling sequences with different pulse frequency $m$. It can be seen how increasing $m$ improves the fidelity, i. e. the suppression of the higher order terms.

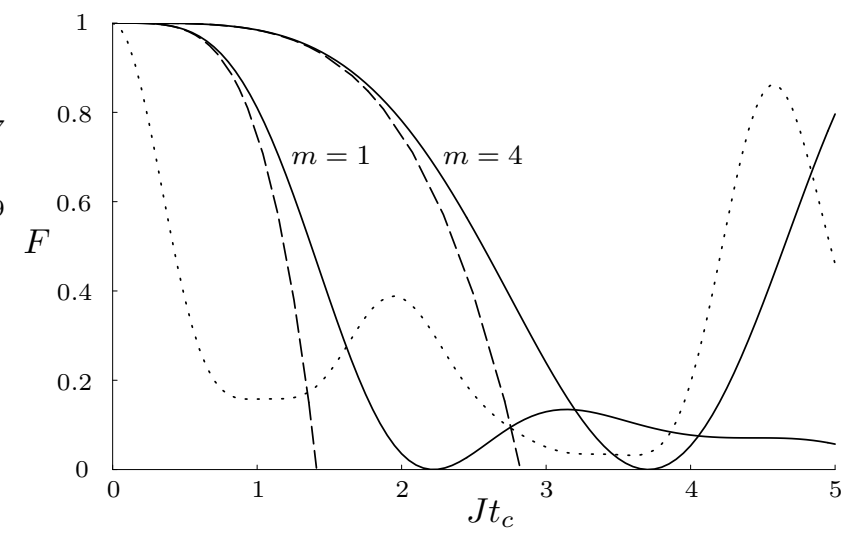

FIG. 1. Decoupling for $N=4$ qubits, $\omega=0$. Shown is the fidelity $F\left(t_{c}\right)$ for free evolution $(\cdots \cdots)$, decoupling with $m=1$ $(-)$ and the corresponding approximation $F=1-(J t)^{4} / 4$ (- - ), decoupling with $m=4$ ( -$)$ and the approximation $F=1-(J t)^{4} / 64(---)$.

\section{DECOUPLING WITH FAULTY GATES}

Up to now we have only considered sequences consisting of perfect pulses, implementing exactly a $\pi$ rotation. This assumption is, of course, not tenable in any real 
physical system. Even for ideal pulses the decoupling is generally not perfect due to higher order terms, but as stated above, these terms can be suppressed by increasing the pulse frequency $m$. Yet with faulty gates this strategy is not necessarily successful, because more errors are then introduced into the system from the outside.

In order to see what happens we study a very simple model, two spins that interact via scalar coupling. We again use sequence (3.8) but now we assume faulty $x$ pulses, where the rotation angle differs from $\pi$ by a small deviation $\delta$, so that

$$
\hat{X}^{(1)}(\delta)=\mathrm{i} \sigma_{x}^{(1)} \cos \frac{\delta}{2}-\hat{1} \sin \frac{\delta}{2},
$$

where $\delta$ is randomly distributed with the probability distribution $w(\delta, \sigma)=(\sqrt{2 \pi} \sigma)^{-1} e^{-\delta^{2} / 2 \sigma^{2}}$. The standard deviation $\sigma$ measures the scatter of the pulses.

As a first case we consider weak coupling, with

$$
\mathcal{H}=-\omega_{z}^{(1)} \hat{\sigma}_{z}^{(1)}-\omega_{z}^{(2)} \hat{\sigma}_{z}^{(2)}+J \hat{\sigma}_{z}^{(1)} \hat{\sigma}_{z}^{(2)} .
$$

Of course, in this case one would not use the above strategy because the higher order terms vanish anyway, and increasing the number of applied pulses will, with certainty, lead to a degraded performance. By induction one can show analytically that after application of $m$ decoupling cycles the fidelity for one of the product basis states $(|00\rangle,|01\rangle,|10\rangle,|11\rangle)$ as initial state is given by

$$
F_{\sigma}(m)=\frac{1}{2}\left(1+e^{-m \sigma^{2}}\right) .
$$

For increasing $\sigma$, i. e. faultier gates, the fidelity decreases as expected.

For the case of strong coupling,

$$
\mathcal{H}=-\omega\left(\hat{\sigma}_{z}^{(1)}+\hat{\sigma}_{z}^{(2)}\right)+J \boldsymbol{\sigma}^{(1)} \cdot \boldsymbol{\sigma}^{(2)},
$$

we have resorted to a numerical calculation of the fidelity after the time $t_{c}$ for the initial state $|01\rangle$, for different $\sigma$ and numbers of repetitions $m$ of base cycles. For each set of parameters we calculated the mean over 1000 realizations with randomly picked deviations $\delta$. Our results are shown in Fig. 2. It turns out that the resulting behavior can be approximated pretty well by the product of the function for $\sigma=0$, approximated by (6.4), with the "damping" resulting for weak interaction (7.3), yielding

$$
F_{\sigma}(m) \approx \frac{1}{2}\left(1+e^{-m \sigma^{2}}\right)\left(1-\frac{1}{400 m^{2}}\right) .
$$

As one can see, we have indeed two competing effects: for small $m$ and $\sigma$ the suppression of the higher order corrections is dominant, whereas for larger $m$ and $\sigma$ the errors introduced by the faulty gates dominate. In particular, there exists for a given $\sigma$ an ideal number of iterations $m$ which yields the maximum fidelity. The corresponding $F_{\max }$ decreases thereby for increasing error. This means that for faulty gates it is not possible anymore to achieve perfect decoupling. This conclusion gets increasingly significant for larger $N$ : then $F_{\max }$ is further reduced by the effect of the additional faulty gates and the rising influence of higher order terms.

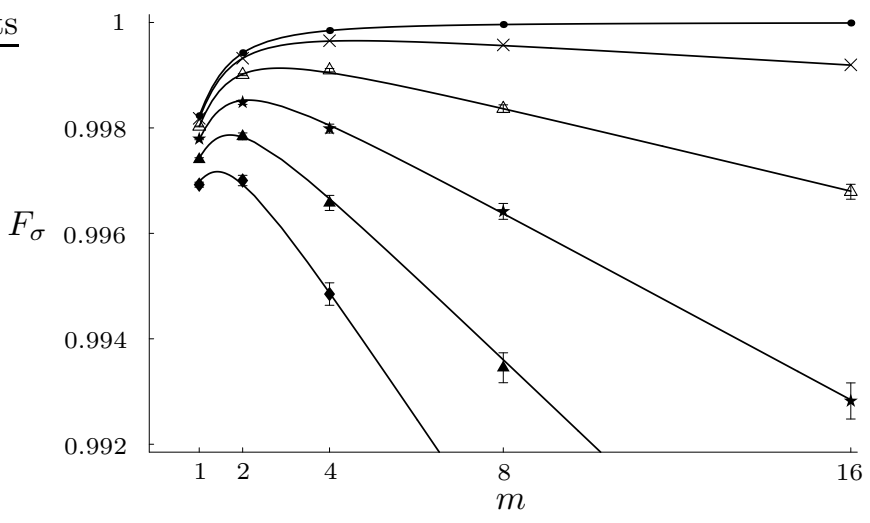

FIG. 2. Decoupling for $N=2$ qubits with faulty gates, strong $J$-coupling. Shown is the fidelity $F_{\sigma}$ at $t_{c}=1 / J$, $J=10 \omega$. The curves correspond to $\sigma=0(\bullet), 0.01(\times)$, $0.02(\triangle), 0.03(\star), 0.04(\boldsymbol{\Delta}), 0.05(\diamond)$. The approximation function is $F_{\sigma}(m)=\frac{1}{2}\left(1+e^{-m \sigma^{2}}\right)\left(1-\frac{1}{400 m^{2}}+\frac{1}{1369 m^{4}}\right)$; with correlation coefficient 0.999996 for $\sigma=0$.

\section{SUMMARY AND CONCLUSIONS}

We have shown that it is possible to generalize refocusing methods to systems of spins coupled by arbitrary two-spin interactions. Thereto spin-selective operations are necessary. We have given a concrete sequence for first order decoupling for $N=2$, and schemes for constructing sequences for $N>2$. These schemes are efficient regarding the needed time, which scales linearly with the size of the system, and the amount of pulses, which scales at most quadratically.

However, in the general case there are higher order terms that must be taken into account. These can be suppressed by increasing the pulse frequency, assuming ideal gate operations. Perfect decoupling for long times could only be achieved by infinitely fast decoupling pulses.

These results hold for the most demanding case of an arbitrary coupling model. Yet for special interaction types, much more efficient schemes are possible. Direct dipolar couplings, for example, can be decoupled using nonselective pulse sequences, that work independent of the system size. For weak scalar coupling all higher order correction terms vanish, so that the decoupling is perfect. The sequences can also be considerably simplified for only partly coupled systems, like a spin chain with nearest neighbor couplings only.

In a real physical system pulses never are ideal. For faulty gate operations perfect decoupling is no longer possible, because of the inaccuracies introduced into the system by the procedure itself. 
The present results are not restricted to the special task of removing all interactions in a spin system. Minor modifications allow specific coupling terms to be turned on or off. Combining on- and off-periods one can even rescale the various parameters within the original Hamiltonian. Generally, a wide range of effective Hamiltonians can thus be invoked. In this sense, complete decoupling is a special case of a quantum simulation [17], where a system is modeled that "does nothing", i. e. any initial state remains unchanged. Such a system is, in general, quite challenging to realize and may thus be considered a serious testing scenario for quantum dynamical control.

\section{ACKNOWLEDGEMENTS}

We thank J. Gemmer, I. Kim, A. Otte, F. Tonner, and T. Wahl for fruitful discussions.

\section{APPENDIX: RECURSIVE CONSTRUCTION OF ORTHOGONAL ARRAYS}

This construction is due to Bose and Bush [15]. Examples can also be found in 14 .

1. Construct an $O A(16 \lambda, 4 \lambda, 4,2), \lambda=2^{u}, u \in \mathbb{N}$, as follows: Take an $M_{4 \lambda}$ [a $\left.D(4 \lambda, 4 \lambda, 4)\right]$; then the $O A$ $A_{0}$ is given by

$$
A_{0}=M_{4 \lambda} \otimes\left(\begin{array}{llll}
I & X & Y & Z
\end{array}\right) .
$$

2. If possible, construct an $O A A_{1}$ with $\lambda_{1}=\lambda / 4$, according to 1 . Repeat each column 4 times, yielding

$$
A_{1}^{\prime}=A_{1} \otimes\left(\begin{array}{llll}
I & I & I & I
\end{array}\right) .
$$

If possible, repeat this step with an $O A A_{2}$ where $\lambda_{2}=\lambda / 4^{2}$, and form an $A_{2}^{\prime}$ by repeating each column $4^{2}$ times, and so on. Append the matrices $A_{1}^{\prime}$, $A_{2}^{\prime}, \ldots$ to $A_{0}$.

3. Add a row consisting of $4 \lambda I$ 's, $4 \lambda X$ 's, and so on.

We now have an $O A(16 \lambda, k, 4,2)$ with $\lambda=2^{u}, u \in \mathbb{N}$, and $k=4 \lambda+4 \lambda_{1}+\cdots+1$ rows, or

$$
k=\frac{\lambda\left(4^{c+1}-1\right)}{4^{c}-4^{c-1}}+1, \quad c=\left\{\begin{array}{ll}
u / 2, & u=0,2, \ldots \\
(u-1) / 2, & u=1,3, \ldots
\end{array} .\right.
$$

The $O A$ (4.2) has been constructed in this way, using $M_{4}$. A $\lambda_{1}=1 / 4$ does not exist, so step 2 was omitted, resulting in $k=4+1=5$. An $O A(32,9,4,2)$ can be constructed using $M_{8}$. For an $O A(64,21,4,2)$ we construct $A_{0}$ using $M_{16}=M_{4} \otimes M_{4}$ and $A_{1}$ using $M_{4}$, so $k=16+4+1=21$.
[1] D. P. DiVincenzo, Science 270, 255 (1995).

[2] D. Loss and D. P. DiVincenzo, Phys. Rev. A 57, 120 (1998).

[3] B. E. Kane, Nature 393, 133 (1998).

[4] N. A. Gershenfeld and I. L. Chuang, Science 275, 350 (1997).

[5] D. G. Cory, M. D. Price, and T. F. Havel, Physica D 120, 82 (1998).

[6] B. Georgeot and D. L. Shepelyansky, Phys. Rev. E 62, 3504 (2000).

[7] N. Linden, H. Barjat, R. J. Carbajo, and R. Freeman, Chem. Phys. Lett. 305, 28 (1999).

[8] D. W. Leung, I. L. Chuang, F. Yamaguchi, and Y. Yamamoto, Phys. Rev. A 61, 042310 (2000).

[9] J. A. Jones and E. Knill, J. Magn. Reson. 141, 322 (1999).

[10] C. P. Slichter, Principles of Magnetic Resonance, 3rd ed. (Springer Verlag, Berlin, 1990).

[11] R. R. Ernst, G. Bodenhausen, and A. Wokaun, Principles of nuclear magnetic resonance in one and two dimensions (Clarendon Press, Oxford, 1987).

[12] U. Haeberlen, High Resolution NMR in Solids: Selective Averaging (Academic Press, New York, 1976).

[13] J. S. Waugh, L. M. Huber, and U. Haeberlen, Phys. Rev. Lett. 20, 180 (1968).

[14] A. S. Hedayat, N. J. A. Sloane, and J. Stufken, Orthogonal Arrays: theory and applications (Springer-Verlag, New York, 1999).

[15] R. C. Bose and K. A. Bush, Ann. Math. Stat. 23, 508 (1952).

[16] D. Jungnickel, Math. Z. 167, 49 (1979).

[17] S. Lloyd, Science 273, 1073 (1996).

\begin{tabular}{|c|c|c|c|c|c|c|c|}
\hline$*$ & $I X$ & $Y \quad Z$ & + & 0 & 1 & 2 & 3 \\
\hline$I$ & $I X$ & $Y \quad Z$ & 0 & 0 & 1 & 2 & 3 \\
\hline$X$ & $X \quad I$ & $Z Y$ & 1 & 1 & 0 & 3 & 2 \\
\hline$Y$ & $Y \quad Z$ & $I \quad X$ & 2 & 2 & 3 & 0 & 1 \\
\hline$Z$ & $Z Y$ & $X \quad I$ & 3 & 3 & 2 & 1 & 0 \\
\hline
\end{tabular}

TABLE I. Multiplication table for the matrices $I, X, Y, Z$ and addition table for $G F(4)$ 